Research Article

\title{
Analysis of Radial Force and Vibration Energy in a Centrifugal Pump
}

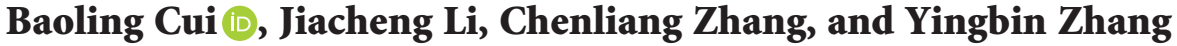 \\ Key Laboratory of Fluid Transmission Technology of Zhejiang Province, Zhejiang Sci-Tech University, Hangzhou, China \\ Correspondence should be addressed to Baoling Cui; blcui@zstu.edu.cn
}

Received 4 December 2019; Revised 1 May 2020; Accepted 26 May 2020; Published 20 July 2020

Academic Editor: Michalis Xenos

Copyright (c) 2020 Baoling Cui et al. This is an open access article distributed under the Creative Commons Attribution License, which permits unrestricted use, distribution, and reproduction in any medium, provided the original work is properly cited.

\begin{abstract}
Vibration is one of the main issues taken into consideration in the design and manufacture of the pump. The radial force and vibration of the impeller induced by fluid in a centrifugal pump were investigated at different flow rates by numerical simulation. The vibrations on the volute were measured by the experiment. The variation trend of the radial displacements of the impeller is consistent with that of the radial forces, and the variation in the radial displacement lags that of the radial force. The vibration energies on the impeller and the volute were analyzed based on root-mean-square (RMS) values in the frequency domain. The distributions of energy loss in the pumps were calculated to determine the total entropy generation (TEG) and entropy generation rate (EGR). The TEG values as calculated are in close accordance with hydraulic loss. The vibration is a result of the poor flow patterns and consequently results in increased energy losses in the pump.
\end{abstract}

\section{Introduction}

Centrifugal pump is a liquid conveying machinery used in petrochemical, aerospace, and chemical industries. Vibration is one of the main issues taken into consideration in the design and manufacture of the pump, especially high-power high-speed centrifugal pumps $[1,2]$. Finite element analysis of fluid-structure interaction (FSI) has become an important method employed to investigate the vibration and deformation of fluid machinery. Many studies have been conducted on FSI in fluid machinery; most of the studies focused on turbines and compressors, while only few studies have investigated pumps [3-6]. Benra [7] carried out numerical and experimental studies on flow-induced vibrations of a single-blade pump impeller and reported good qualitative agreement between the FSI calculation and measurement. Katsutoshi et al. [8] evaluated the characteristics of a mixed-flow pump using FSI simulation and the predicted stresses were in good agreement with experimental values within $-11 \pm 6 \%$ prediction accuracy. Pei et al. [9] calculated the deformation and stress in the impeller of an axial-flow pump with two-way passage using FSI simulation and found that the deformation and stress significantly decreased as the flow rate increased. Schneideret et al. [10] numerically evaluated the deformation and stress in impellers of a multistage pump at different operating conditions using FSI simulations. The hydraulic exciting forces generated by unsteady flow are the main factors responsible for vibration and noise in the pump. Pei et al. [11] investigated the hydrodynamic force and flowinduced vibrations of a single-blade sewage water pump impeller at design and off-design flow rates using numerical and experimental methods. Although FSI problems have received significant research interest, only few researches have been carried out to experimentally validate complex FSI problems. In some cases, the deformations can be neglected or are difficult to be measured for moving or rotating structures [12]. Also, the energy loss caused by unsteady flow has a certain correlation with the vibration. Ren et al. [13] used entropy generation rate (EGR) to compare the energy loss in the multistage pump with and without the guide ring. Jia et al. [14] studied the internal instability characteristics of centrifugal pump based on entropy production and vibration energy and found that the internal flow loss has the same variation rule as the vibration energy. 
In this study, the radial force and vibration of the impeller induced by fluid in a centrifugal pump were investigated using ANSYS 18.0 commercial software. The radial force and vibration displacement of the impeller were determined at different flow rates. The performance and vibration experiments were conducted on the centrifugal pump. The relationship between energy loss and vibration energy was analyzed based on the entropy generation method.

\section{Geometrical Model and Numerical Method}

The design parameters of the centrifugal pump used in this study are as follows: flow rate $Q_{\mathrm{d}}=18 \mathrm{~m}^{3} / \mathrm{h}$, head $H=18 \mathrm{~m}$, and rotating speed $n=2950 \mathrm{r} / \mathrm{min}$. The specific speed is $23.8 \mathrm{~m}^{0.75} \cdot \mathrm{s}^{-1.5}$ calculated by equation $n_{s}=\left(n \times \sqrt{\mathrm{Q}} / H^{0.75}\right)$. The main geometrical parameters are as follows: the inlet diameter of the pump is $65 \mathrm{~mm}$, the outlet diameter of the pump is $50 \mathrm{~mm}$, the impeller diameter is $130 \mathrm{~mm}$, and the number of blades is 5 .

A three-dimensional model of the entire flow field was established using Pro/E, including the inlet, impeller, volute, impeller side chamber, outlet, and annular seal at the impeller inlet. The computational domains for fluid and solid are shown in Figure 1, where Figure 1(a) presents the threedimensional flow domain, Figure 1(b) presents the twodimensional section drawing, and Figure 1(c) presents the solid domain and monitor positon of vibration.

The flow calculation domain was meshed with structural grid generated using ICEM-CFD commercial software. The solid domain including the shaft and impeller was meshed with ANSYS software. The computational grids for fluid and solid domain are shown in Figure 2, where Figures 2(a)-2(c) present the grids in the computational fluid domain and Figure 2(d) presents the grids for solid domain.

A grid number test of the computational flow domain was conducted to validate grid independence in the numerical simulation, as shown in Figure 3. The elements of the pump were 1891586, 3301394, 4402428, and 6381025. To ensure a comprehensive consideration of calculation accuracy and efficiency, the total number of elements used in the numerical simulation was 4402428. The elements for the inlet, impeller, volute, outlet, and impeller side room were 490930, 1391721, 1162718, 423291, and 933768, respectively. The grids near the solid wall were refined to satisfy the applicability of the turbulence model. The layers number in the clearance is ten for annular seal of impeller inlet. And it is fifteen for the chamber room of the impeller. The mean $y+$ value of the impeller was 9.99915. For the solid region, there were 88,940 elements and 15,736 nodes, which were meshed with nonstructural grids.

The temporal discretization accuracy depends on the time step size, which is of great significance to capture the pressure fluctuation. The influence of the time step was also investigated in this study. The impeller rotates at time steps of $1^{\circ}, 1.5^{\circ}, 2^{\circ}, 3^{\circ}$, and $5^{\circ}$. Figure 4 shows the results of fast Fourier transformation (FFT) of the pressure fluctuation near the volute tongue for the five time steps. It can be observed that the main peak occurred at $245.6 \mathrm{~Hz}$, corresponding to the blade passing frequency. When a smaller time step size was used, the peaks occurred at the same frequency with a minor difference in magnitude. The results indicate that impeller rotation at every $1.5^{\circ}$ is sufficient.

In this study, the vibration of the impeller was investigated based on one-way coupling FSI simulations using CFX and Workbench in ANSYS 18.0. The periodically stable results in unsteady flow simulation were used as initial values for FSI simulation. For the fluid region, the SST (shear stress transport) $\mathrm{k}-\omega$ turbulence model was used in numerical simulation for unsteady calculation and the transient rotor-stator model was applied to couple the interface between the rotating and static domains. The velocity inlet was set as the inlet boundary condition, while the opening was set as the outlet boundary condition. Because the rotating speed of the impeller was $2950 \mathrm{r} / \mathrm{min}$, each time step $\Delta t=0.000084746 \mathrm{~s}$. For the solid region, the displacements were calculated based on the FSI simulation using the transient structure modules of the Workbench in ANSYS 18.0. The Hilber-Hughes-Taylor (HHT) method and damping effect were applied in time integration of the transient dynamic equation and the transient analysis, respectively. The contacting fluid surfaces of the impeller and shaft were set as the fluid-structure coupling interface. In each time step, the fluid load was transferred to the structure through the interface. Structural steel with density of $7850 \mathrm{~kg} / \mathrm{m}^{3}$, elastic modulus of $200 \mathrm{GPa}$, and Poisson's ratio of 0.3 was selected as material for the solid region.

\section{Results and Discussion}

3.1. External Performance of Experiment and Simulation. The external characteristics of the head and efficiency of the centrifugal pump obtained from the experiment are shown in Figure 5, and these were compared with those obtained from the unsteady numerical simulation. It can be observed in Figure 5 that the simulated head and efficiency of the centrifugal pump were slightly higher than the experimental values, but both have the same trend. The experimental and simulation values of the head and efficiency at the design flow rate were $H_{\mathrm{e}}=17.77 \mathrm{~m}$ and $\eta_{\mathrm{e}}=58.87 \%$ and $H_{\mathrm{s}}=18.40 \mathrm{~m}$ and $\eta_{\mathrm{s}}=63.17 \%$, respectively. During simulation, hydraulic smooth surfaces were applied for the pump components. The measured efficiency includes mechanical losses, which were not considered in the simulation. This accounts for some of the observed differences between experimental and simulation results.

\subsection{Static Pressure Distribution in Centrifugal Impeller.}

The static pressure distributions in the middle section of the impeller are shown in Figure 6 at $0.6 Q_{\mathrm{d}}, 0.8 Q_{\mathrm{d}}, Q_{\mathrm{d}}$, and $1.2 Q_{\mathrm{d}}$. From Figure 6, it can be seen that the static pressure in the impeller increases from the inlet to outlet. The pressure is larger on the pressure side than that on the suction side of the blade. The biggish pressure in the impeller is at outlet of the blade and it is relatively greater at $0.6 Q_{\mathrm{d}}$ than that $0.8 Q_{\mathrm{d}}, Q_{\mathrm{d}}$, and $1.2 Q_{\mathrm{d}}$. According to the law of performance curve, the head at $0.6 Q_{\mathrm{d}}$ is larger than that at $0.8 Q_{\mathrm{d}}, Q_{\mathrm{d}}$, and $1.2 Q_{\mathrm{d}}$. This may result in the biggish radial force at $0.6 Q_{\mathrm{d}}$. 


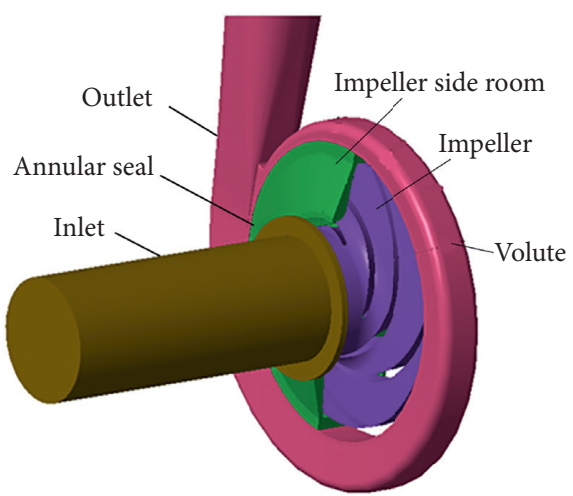

(a)

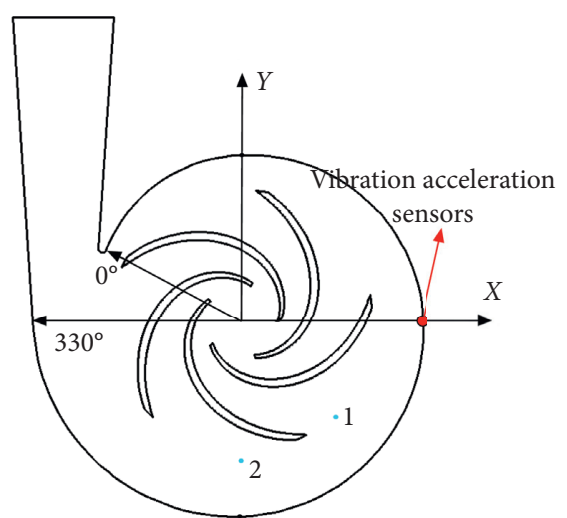

(b)

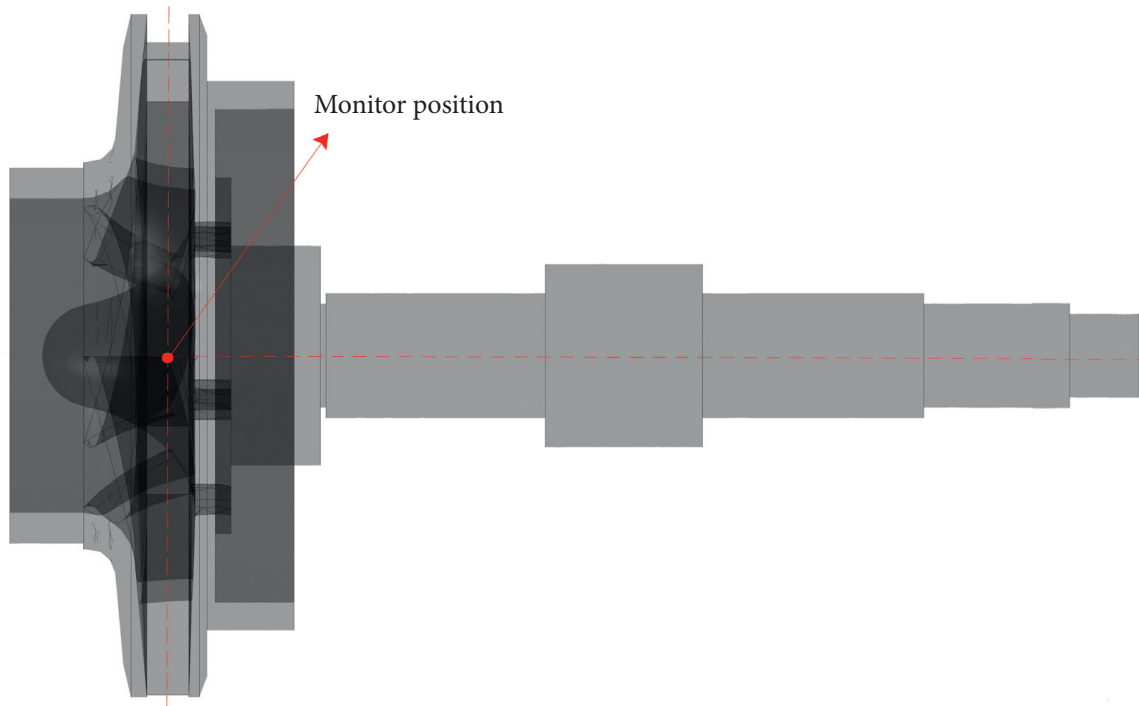

(c)

Figure 1: Computational domains. (a) Three-dimensional flow domain. (b) Two-dimensional section. (c) Monitor position of solid domain.

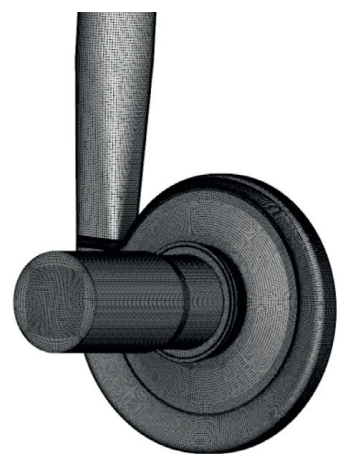

(a)

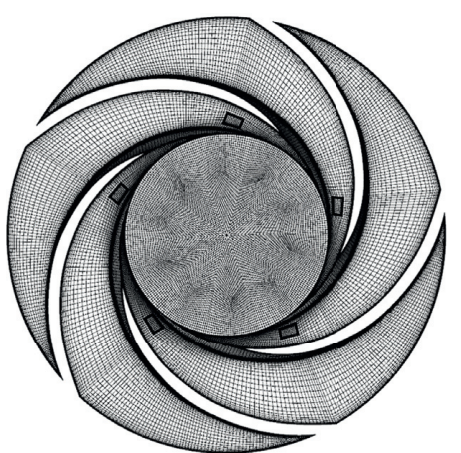

(b)
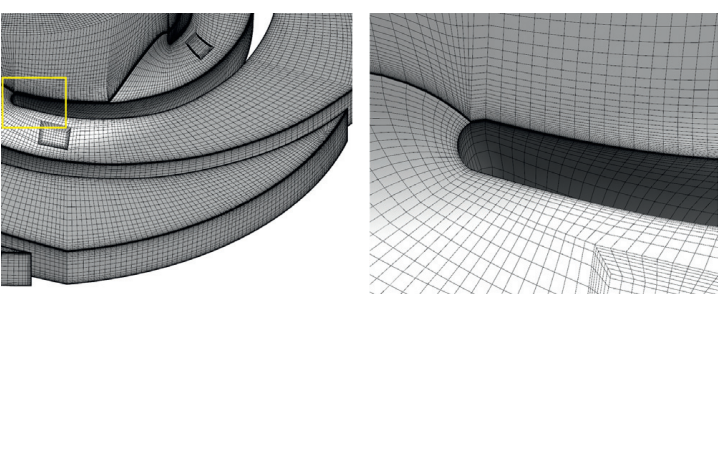

(c)

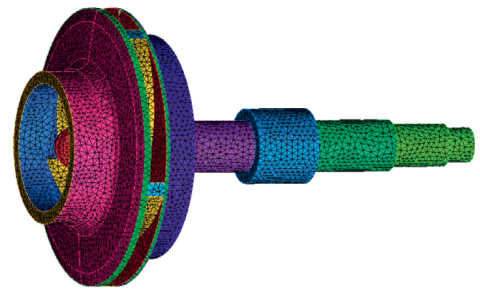

(d)

Figure 2: Computational grids. (a) Whole fluid domain. (b) Impeller. (c) Local refinement for impeller. (d) Solid domain. 


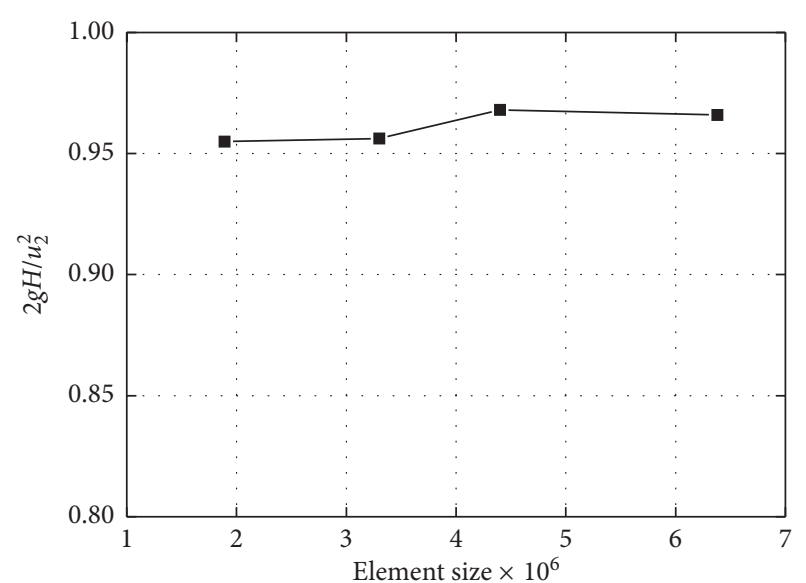

FIGURE 3: Grid independence verification.

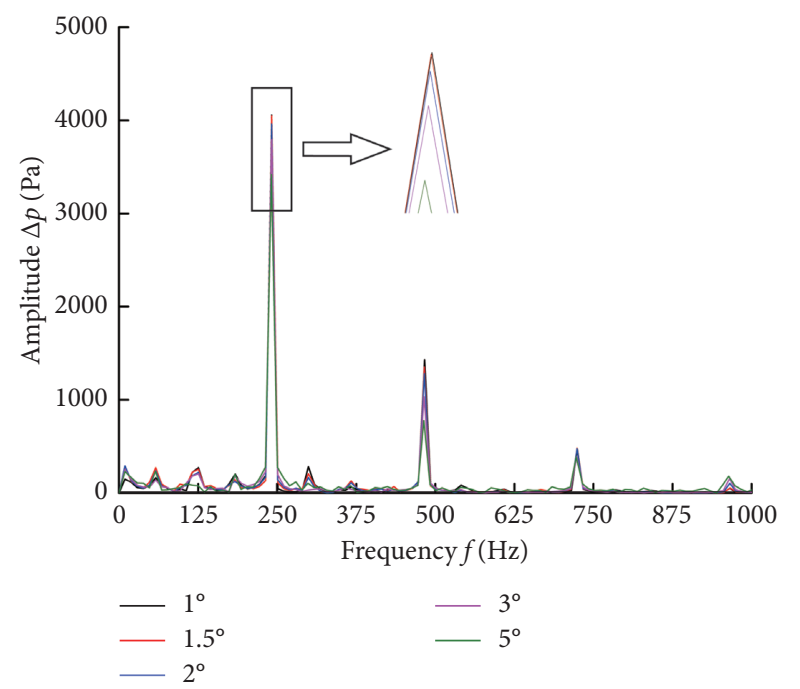

Figure 4: Fast Fourier transformation of the pressure fluctuation for the five time steps.

The static pressure coefficient $C \mathrm{p}$ (defined by $\left(p-p_{\text {in }}\right) /$ $\left.\left(\rho u^{2} / 2\right)\right)$ of monitoring points 1 and 2 near the outlet of the impeller marked in Figure 1(b) is shown in Figure 7 at $0.6 Q_{\mathrm{d}}$, $0.8 Q_{\mathrm{d}}, Q_{\mathrm{d}}$, and $1.2 Q_{\mathrm{d}}$. From Figures $7(\mathrm{a})$ and $7(\mathrm{~b})$, it can be seen that the static pressures fluctuate periodically in one rotating cycle. The maximum peak values of static pressure for monitoring points 1 and 2 basically decrease with the increasing of the flow rate, which are in accordance with the law of head change at different flow rates. From Figures 7(c) and $7(\mathrm{~d})$, it can be seen that the dominant frequencies of pressure fluctuation both are at blade passing frequency for the two monitoring points. Also there are fluctuation amplitudes at blade passing frequency multiplication. The fluctuation amplitudes of monitoring points at $0.6 Q_{\mathrm{d}}$ are larger than that at other flow rates.

3.3. Radial Force and Vibration on Centrifugal Impeller. Radial forces generated by unsteady flow are one of the important factors that affect operation stability, and these result in pump vibration. So the radial forces and vibrations

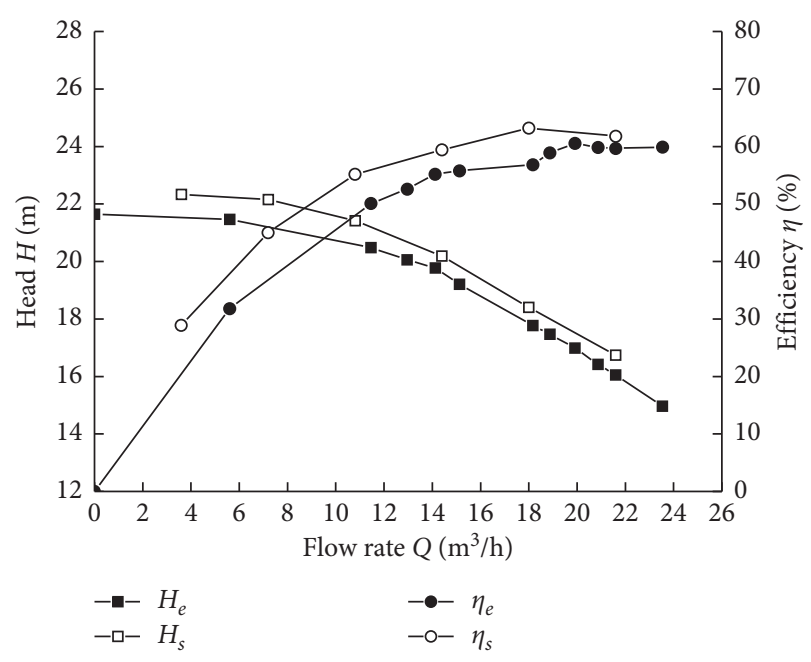

FIGURE 5: External characteristics of the centrifugal pump.

on the impeller were investigated at $0.6 Q_{\mathrm{d}}, 0.8 Q_{\mathrm{d}}, Q_{\mathrm{d}}$, and $1.2 Q_{\mathrm{d}}$.

3.3.1. Radial Force on the Impeller. The time domain diagrams of the radial force in the $X$-direction, $Y$-direction, and resultant force on the impeller are shown in Figure 8 at $0.6 Q_{\mathrm{d}}, 0.8 Q_{\mathrm{d}}, Q_{\mathrm{d}}$, and $1.2 Q_{\mathrm{d}}$.

As shown in Figure 8, the radial forces are periodic and vary with the time. The minimum radial force occurred at the design flow rate, while the radial forces increased, when the pump runs at off-design conditions. The radical force increased as the flow rate decreased. The radial forces and their fluctuations were relatively large at a flow rate of $0.6 Q_{d}$. Theoretically, the velocity and pressure of fluid in the spiral volute around the impeller are uniform and axisymmetric, and the radial force on the impeller is zero at the design flow rate. In fact, the interaction between the volute tongue and impeller blade destroys the axisymmetric distribution of pressure along the circumference of the impeller. This results in the radial force on the impeller at the design flow rate. When the flow rate is less than the design flow rate, the fluid flow is equivalent to that in a diverging pipe relative to the design flow rate. The velocity of fluid in the volute becomes increasingly low from the tongue to the diffusion pipe; however, the pressure gradually increases. At a high flow rate, the opposite trend occurs relative to that of a low flow rate, which results in opposing directions of the radial force under the two conditions. As shown in Figures 8(a) and 8(b), the directions of the $X$-direction and $Y$-direction radial forces at flow rates of $0.6 Q_{\mathrm{d}}$ and $0.8 Q_{\mathrm{d}}$ are opposite to that at a flow rate of $1.2 Q_{d}$ for most time. As shown in Figure 8(c), the variation trend of the resultant force is similar at different flow rates, and five peaks and five troughs occurred in one cycle, which is the same as the number of blades. Owing to the interaction between the blade and tongue, the internal pressure fluctuates, which leads to variations in the radial forces. The radial forces increased after the troughs and reached their peaks, when the blades sweep through the tongue. A possible explanation is that the pressure 


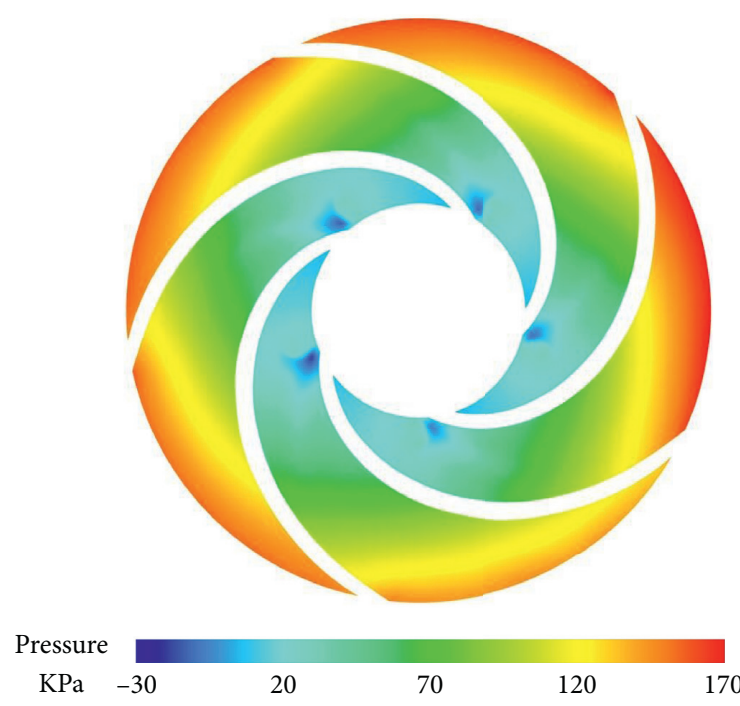

(a)

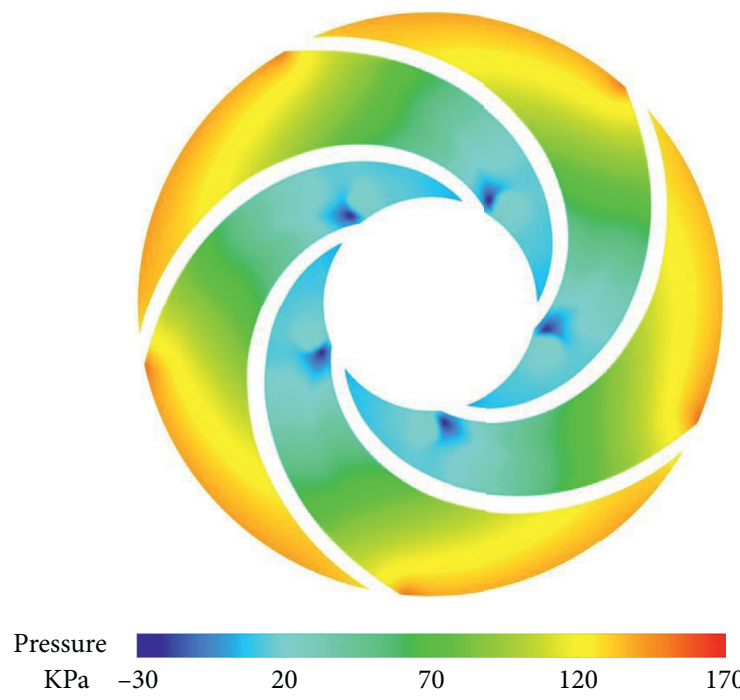

(c)
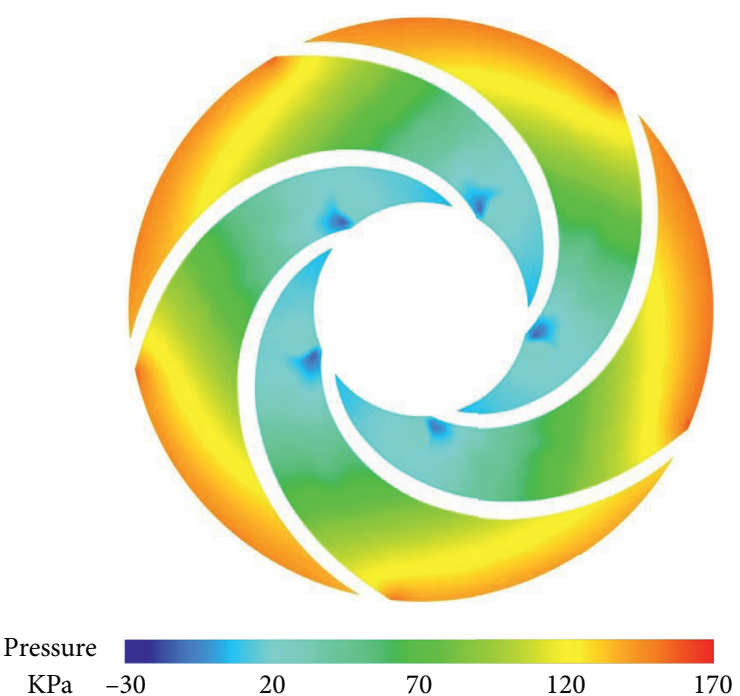

(b)

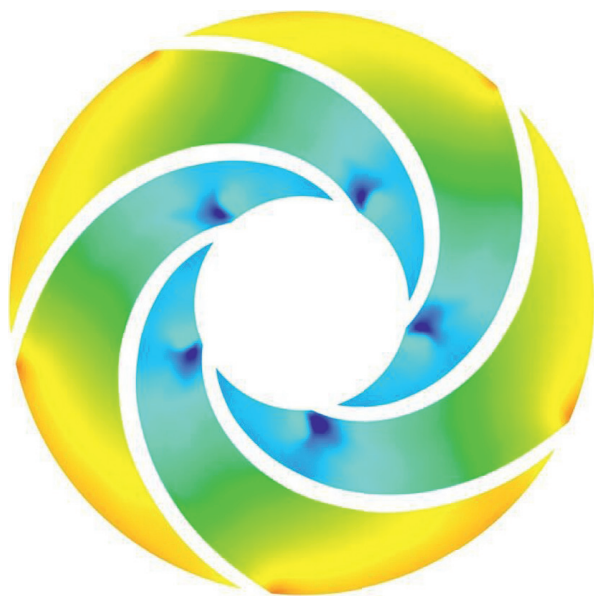

Pressure

$\mathrm{KPa}-30$

20

70

120

170

(d)

FIGURE 6: Static pressure in the impeller. (a) $0.6 Q_{\mathrm{d}}$. (b) $0.8 Q_{\mathrm{d}}$. (c) $Q_{\mathrm{d}}$. (d) $1.2 Q_{\mathrm{d}}$.

fluctuation near the tongue increases as the distance between the tongue and blade decreases. The unbalance of the circumferential pressure leads to a higher radial force on the impeller. Then, the radial forces rapidly decreased as the blades swept through the tongue. After passing the trough, pressures near the volute tongue gradually recovered in the pump and the radial forces reached their peak once again.

3.3.2. Vibration Displacement of the Impeller. The vibration displacement of the impeller was monitored at the intersection point between the central line of the rotating shaft and blade exit width. The vibration displacements in the $X$ - and $Y$-directions and vibration displacement vector of the centrifugal impeller are shown in Figures 9(a)-9(c) at four different flow rates of $0.6 Q_{\mathrm{d}}, 0.8 Q_{\mathrm{d}}, 1.0 Q_{\mathrm{d}}$, and $1.2 Q_{\mathrm{d}}$. In Figures $9(\mathrm{a})$ and 9 (b), the horizontal axis indicates the time, while the longitudinal axis indicates the impeller displacement. Fluctuations in the radial displacement in the $X$ - and $Y$-directions are all periodic. Out of the four flow rates, the impeller displacements were lowest at the design flow rate. The variation trends of the radial displacement in the $X$ - and $Y$-directions were consistent with those of the radial forces on the impeller. The positive or negative direction of the $X$-direction and $Y$ direction radial displacement at flow rates of $0.6 Q_{\mathrm{d}}$ and $0.8 Q_{\mathrm{d}}$ was opposite that at a flow rate of $1.2 Q_{\mathrm{d}}$ for most of the time, which decreased compared to the range of radial forces in the corresponding direction. The reason is that the variation in the radial displacement lags the radial force. The positivedirection displacement in the $X$-direction lies at the first and fourth quadrants, while the positive-direction displacement in the $Y$-direction lies at the first and second quadrants in Figure 9(c). As shown in Figure 9(c), the radial displacements 


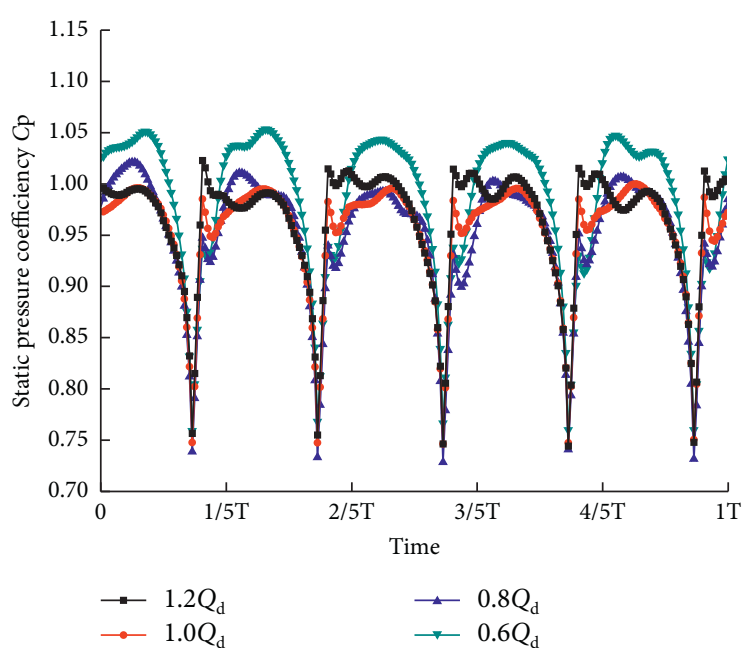

(a)

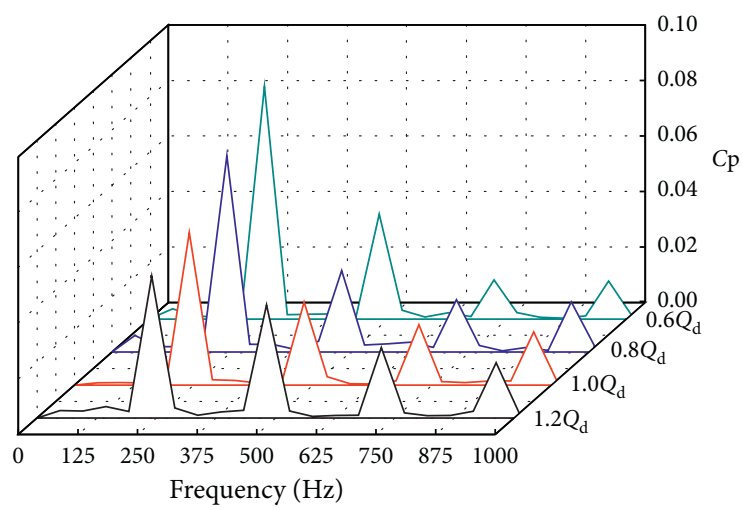

(c)

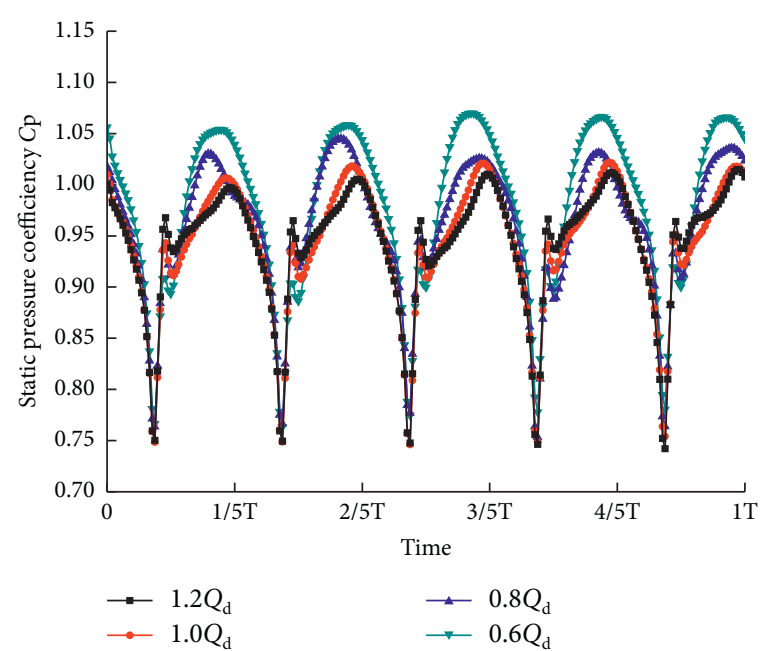

(b)

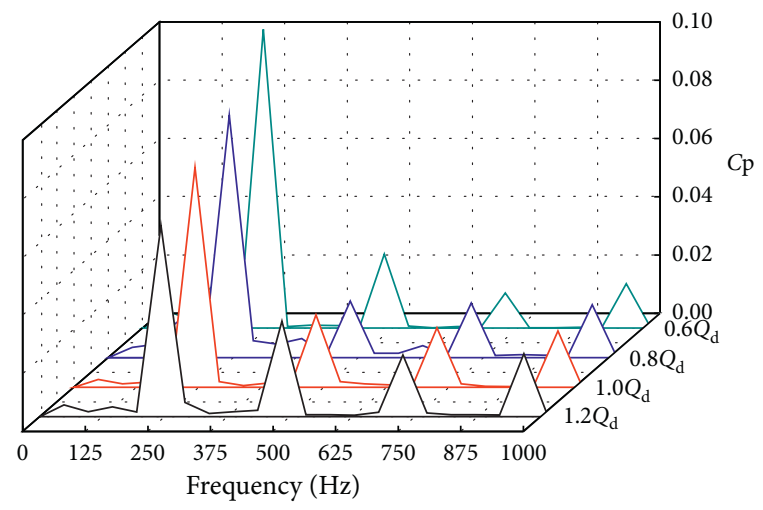

(d)

Figure 7: Pressure fluctuation of monitoring point. (a) Time domain of monitoring point 1. (b) Time domain of monitoring point 2. (c) Frequency domain of monitoring point 1. (d) Frequency domain of monitoring point 2.

in the $X$ - and $Y$-directions increased, when the pump runs at off-design conditions and the maximum vibration displacement occurred at a flow rate of $0.6 Q_{\mathrm{d}}$. The first and fourth quadrants of the displacement were larger than the other quadrants at flow rates of $0.6 Q_{\mathrm{d}}$ and $0.8 Q_{\mathrm{d}}$. The displacements of the second and third quadrants at a flow rate of $1.2 Q_{\mathrm{d}}$ were larger than those of the other quadrants.

The frequency domains of the vibration acceleration of the impeller are shown in Figure 10. The amplitudes of the peak in the $X$ - and $Y$-directions are mainly at the same, fourfold, sixfold, and ninefold shaft rotating frequency and same, twice, and thrice blade passing frequency. The amplitudes of the peak at $Q_{\mathrm{d}}$ are less than that of other flow rates, when the frequency is less than triple blade passing frequency. They are larger at blade passing frequency and frequency multiplication. The rotor-stator interaction between the blade and tongue has an important influence on vibration acceleration.

3.4. Vibration Experiment on the Volute and Vibration Energy. Unsteady flow can cause severe energy loss in the centrifugal pump, where vibration energy as one of the main energy losses will severely affect the efficiency of the centrifugal pump.

3.4.1. Vibration Experiment on the Volute. The pump is a magnetic force pump and vibration measurement cannot be conveniently carried out on the shaft. Thus, the vibration acceleration sensors indicated in Figure 1(b) were installed on the volute in the $X$ - and $Y$-directions. The frequency range was $1-10 \mathrm{kHz}$ and the mounted resonance frequency was $32 \mathrm{kHz}$.

The vibration acceleration on the volute of the pump was measured at four different flow rates. The frequency domains of the vibration acceleration in the $X$ - and $Y$-directions are shown in Figures 11(a) and 11(b), respectively. It can be observed in Figure 11 that there are peaks at $49.2 \mathrm{~Hz}$, $147.5 \mathrm{~Hz}, 245 \mathrm{~Hz}$, and $492.5 \mathrm{~Hz}$ in the $X$ - and $Y$-directions, and these are the same and thrice the shaft rotating frequency and the same and twice the blade passing frequency, respectively. In general, the amplitude of vibration acceleration was relatively high at the shaft rotating frequency and at twice the blade passing frequency. In addition to vibrations induced by the fluid, there were also mechanical 


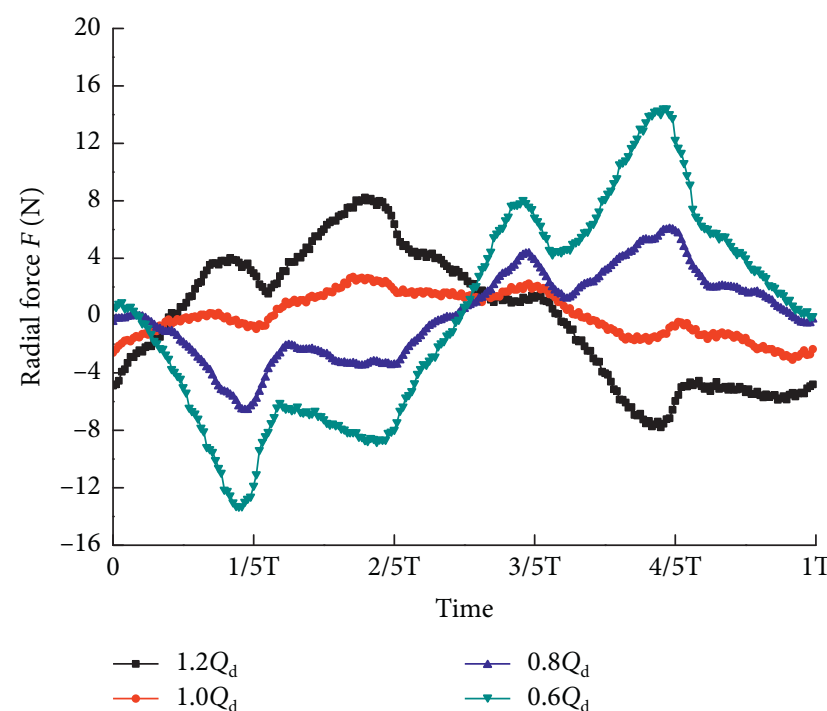

(a)

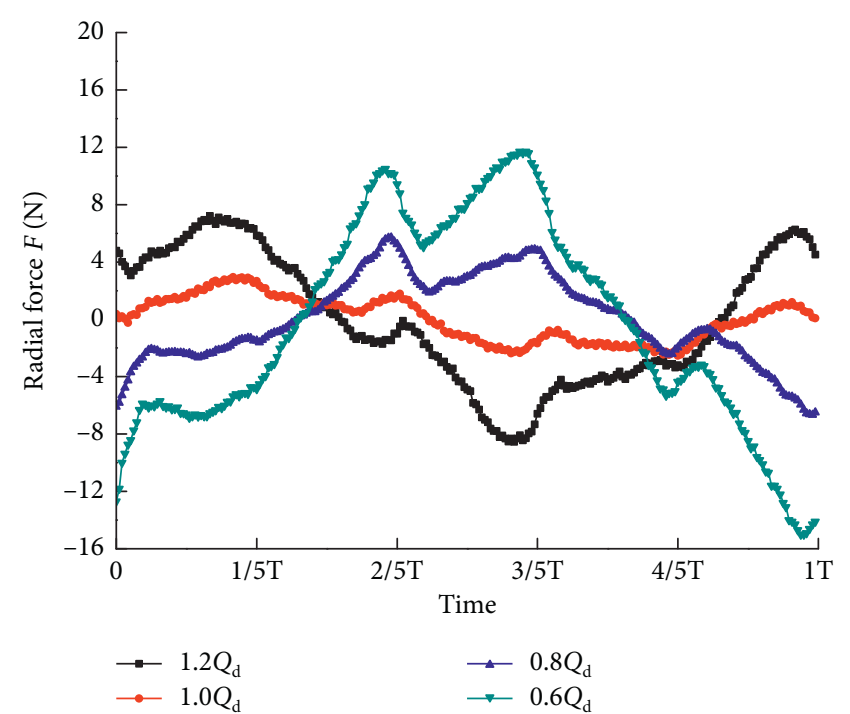

(b)

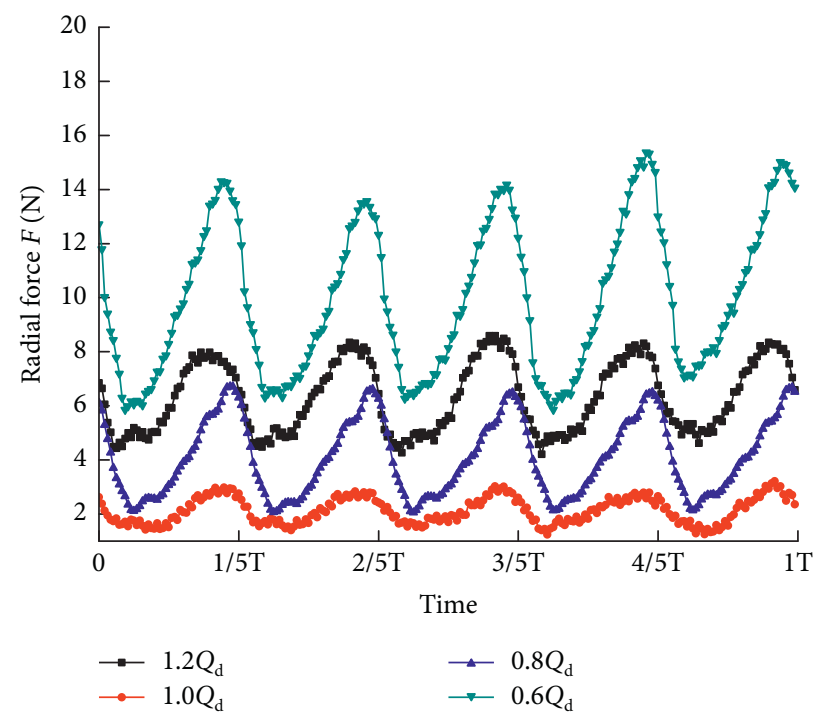

(c)

Figure 8: Radial forces on the impeller. (a) X-direction radial forces. (b) Y-direction radial forces. (c) Resultant forces.

vibrations during the experiment. Thus, the frequency domain of experimental results was more populated, because the pump is vertically installed under the platform and this is heavy and fixed on the base. Also the motor is mounted on the platform. The installation mode makes the vibration of volute very small. The most of dominant frequencies for vibration acceleration on the volute are at shaft frequency. But the most of dominant frequencies for vibration acceleration on the impeller are at blade passing frequency, which is same as the dominant one of pressure fluctuation for the monitoring point at the impeller outlet.

3.4.2. Vibration Energy Analyses. To evaluate the varying trend of vibration energy in the frequency band, RMS method is applied to deal with discrete peaks at different frequencies, as defined in the following equation:

$$
\begin{aligned}
\bar{A} & =\frac{\sum_{i=1}^{n} A_{n}}{n}, \\
\mathrm{RMS} & =\sqrt{\frac{\sum_{i=1}^{n}\left(A_{n}-\bar{A}\right)^{2}}{n}},
\end{aligned}
$$

where $A_{n}$ is the amplitude of the vibration acceleration at each frequency and $n$ is the number of frequency points in each specific frequency band.

Figure 12 presents the vibration energy of impeller and volute at different flow rates. The amplitude of the vibration acceleration on the impeller and volute mainly concentrated at the frequency range of $0-1000 \mathrm{~Hz}$. From $0.6 Q_{\mathrm{d}}$ to $1.0 Q_{\mathrm{d}}$, the vibration energy of impeller and volute decreases with the flow rate and reaches a larger value at $0.6 Q_{\mathrm{d}}$. And it is minimum at the design condition. And it increases at 


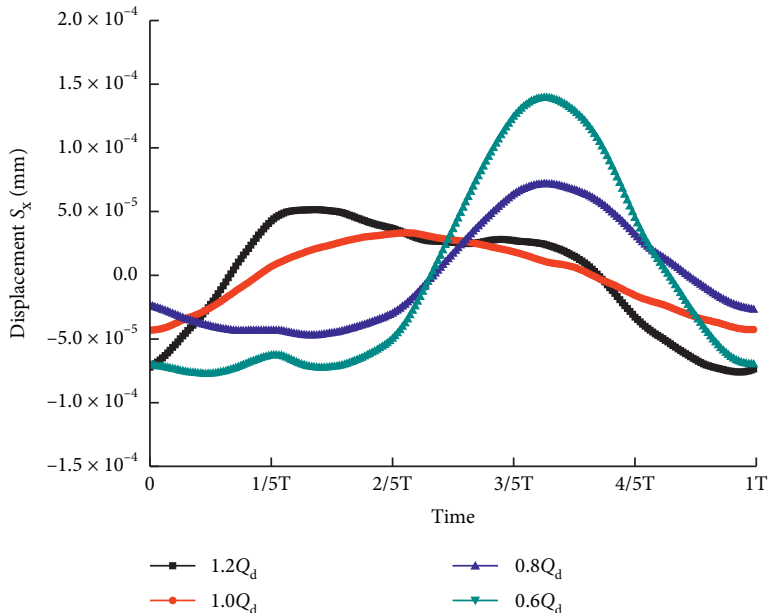

(a)

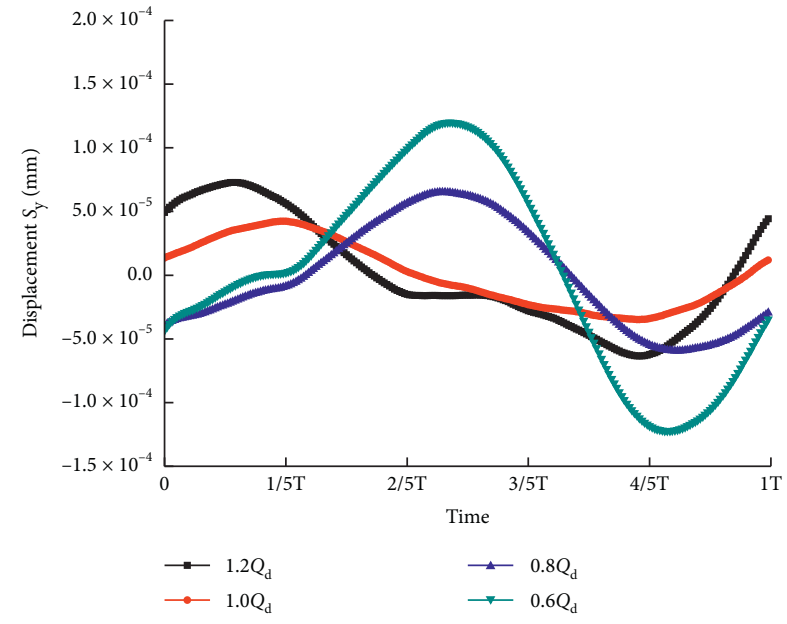

(b)

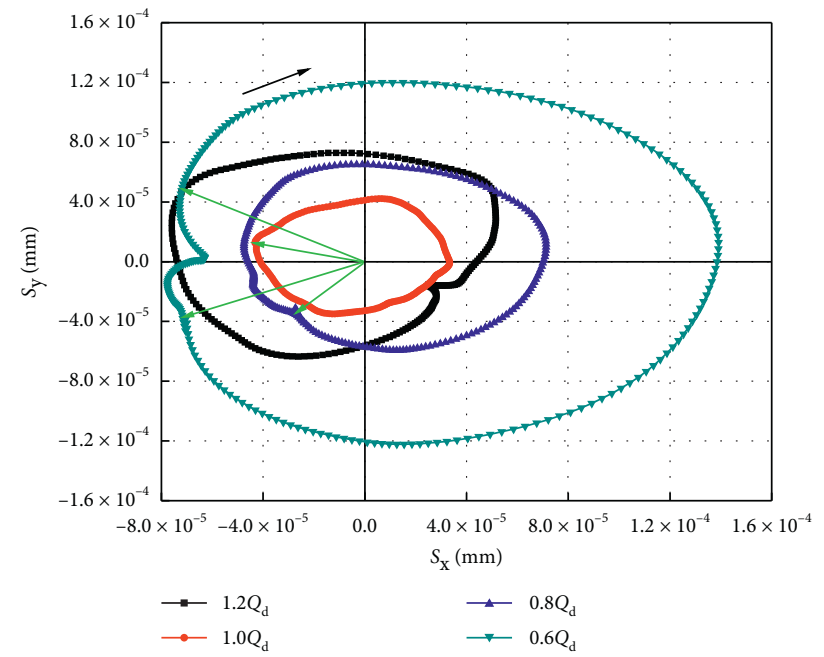

(c)

Figure 9: Vibration displacement of the impeller. (a) X-direction displacement. (b) Y-direction displacement. (c) Vibration displacement vector.

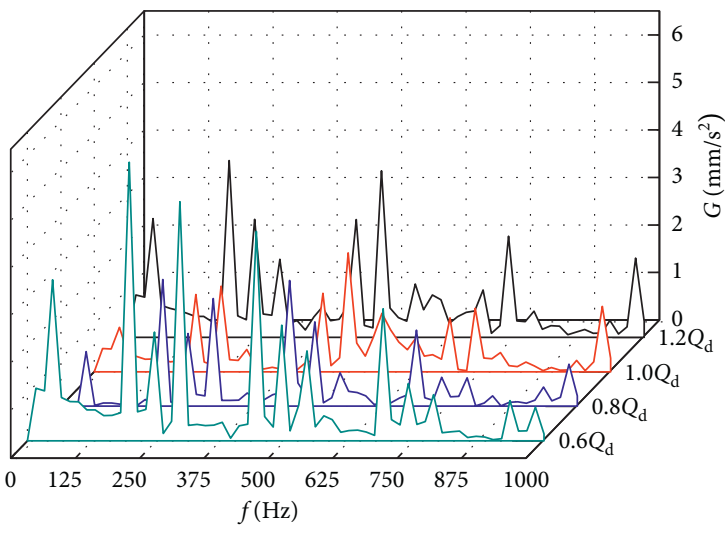

(a)

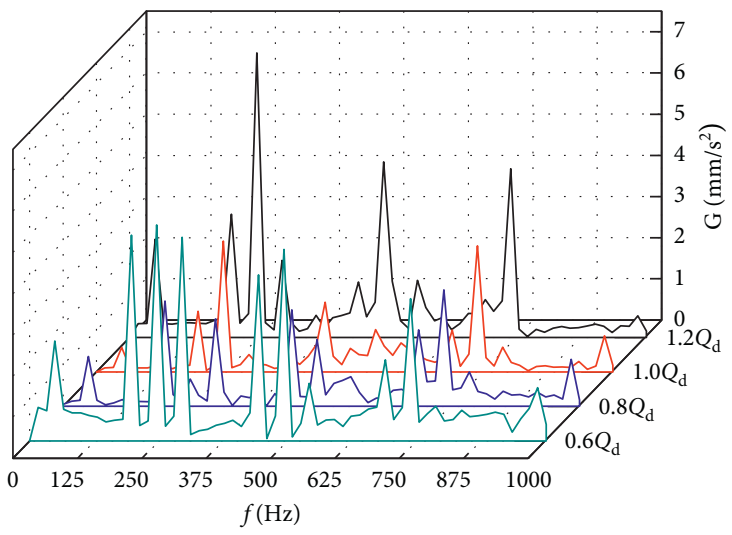

(b)

FIGURE 10: Frequency domains of the vibration acceleration of the impeller. (a) X-direction. (b) Y-direction. 


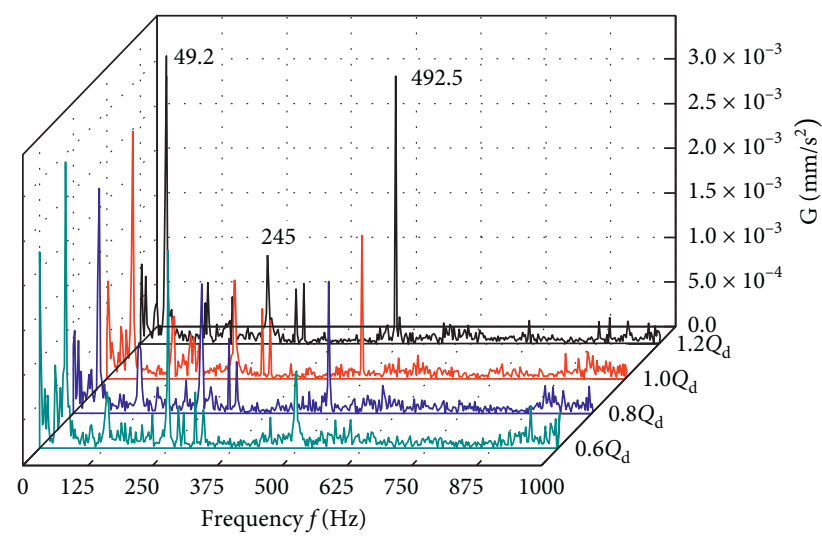

(a)

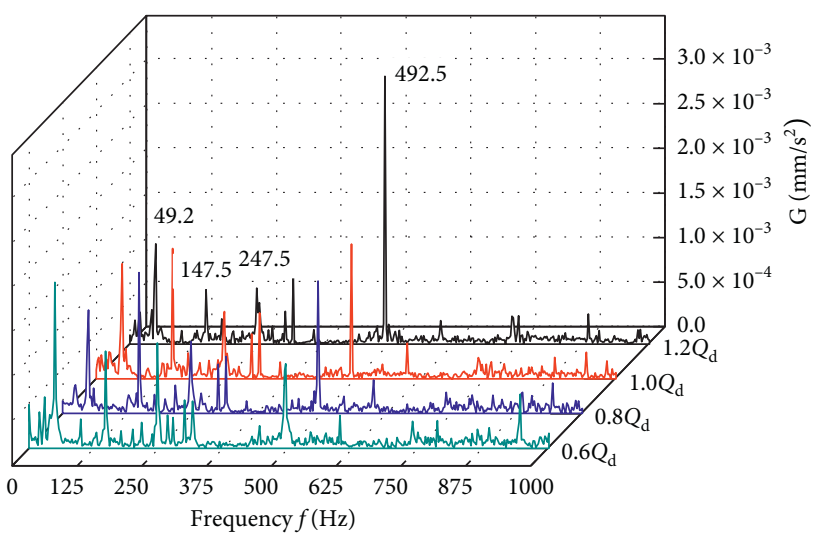

(b)

FIGURE 11: Frequency domains of the vibration acceleration on the volute. (a) $X$-direction. (b) $Y$-direction.

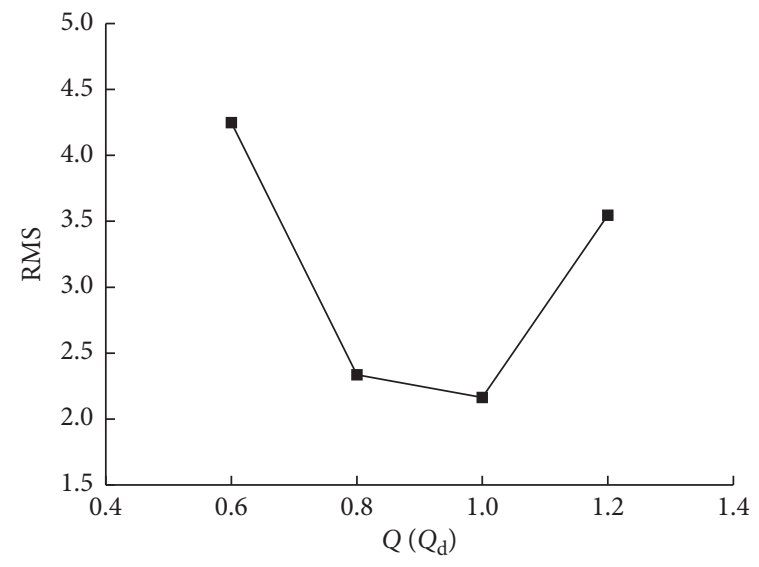

(a)

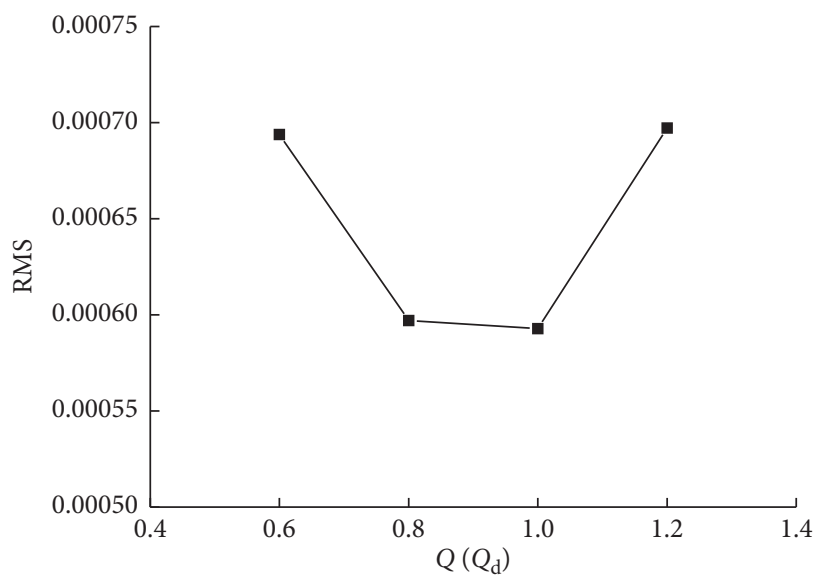

(b)

FIgURE 12: Vibration energy of the impeller and volute at different flow rates. (a) Impeller. (b) Volute.

$1.2 Q_{\mathrm{d}}$ with the increasing of flow rate. Under off-design flow rates, the inflow angle attack will change with the increase or decrease of the flow rates, which could cause vortex shedding from the surface of the blade. The unsteady flow has a significant effect on the pump vibration under off-design conditions.

3.5. Energy Loss of Centrifugal Pump. The energy dissipation caused by the irreversible conversion of the available energy to unavailable energy is mainly manifested by the increase or decrease of entropy generation. The local entropy generation rate in the flow field is defined as follows [15]:

$$
\begin{aligned}
\dot{S}_{D}^{\prime \prime \prime}= & \frac{2\left(\mu+\mu_{t}\right)}{T}\left[\left(\frac{\partial u}{\partial x}\right)^{2}+\left(\frac{\partial v}{\partial y}\right)^{2}+\left(\frac{\partial w}{\partial z}\right)^{2}\right] \\
& +\frac{\left(\mu+\mu_{t}\right)}{T}\left[\left(\frac{\partial u}{\partial x}+\frac{\partial v}{\partial y}\right)^{2}+\left(\frac{\partial u}{\partial z}+\frac{\partial w}{\partial y}\right)^{2}+\left(\frac{\partial v}{\partial z}+\frac{\partial w}{\partial y}\right)^{2}\right], \\
\mathrm{EGR}= & \dot{S}_{D}^{\prime \prime \prime}=\dot{S}_{\bar{D}}^{\prime \prime \prime}+\dot{S}_{D^{\prime}}^{\prime \prime \prime} .
\end{aligned}
$$

Assuming an isothermal flow at a temperature $T, u, v$, and $w$ are the time-averaged velocity components, $\mu$ is the eddy viscosity, and $\mu_{t}$ is the dynamic viscosity. $\bar{D}$ is the contributions due to the time-averaged movement; $D^{\prime}$ is the fluctuations of the velocity field. However, in the calculation of the Reynolds time-average method, the entropy generation caused by the fluctuations of the velocity field is not available. Kock [16] and Mathieu [17] proposed to correlate $\varepsilon$ or $\omega$ in the turbulence model with the entropy generation by the fluctuations of the velocity field. In the calculation of the SST $k-\omega$ turbulence model, the local entropy generation due to the fluctuations of the velocity field can be expressed as

$$
\dot{S}_{D^{\prime}}^{\prime \prime \prime}=\beta \frac{\rho \omega k}{T},
$$

$k$ and $\omega$ are the turbulent kinetic energy and turbulent vortex frequency in the turbulence model, respectively. The constant $\beta=0.09$. The total entropy generation (TEG) is the volume integration of the specific entropy generation rate, which can be expressed as 


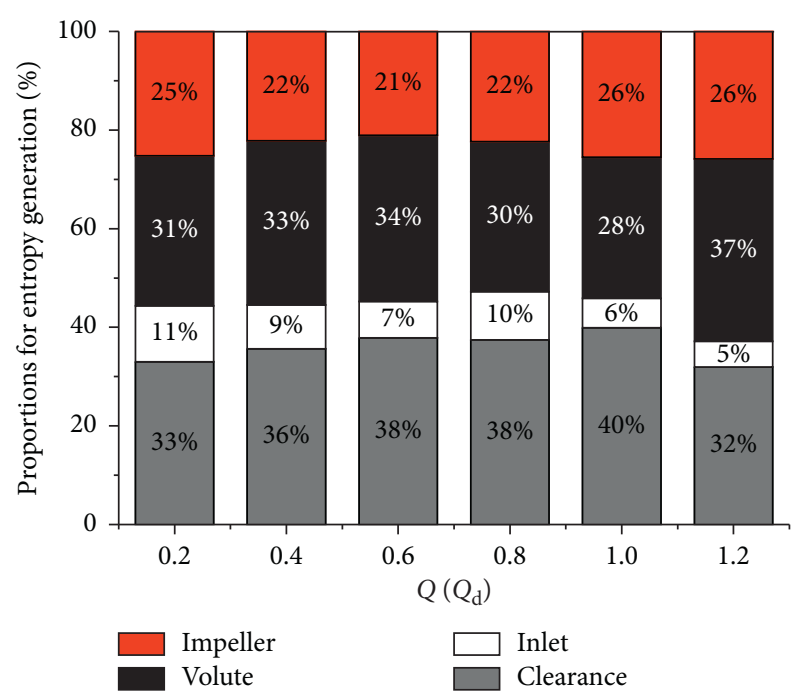

(a)

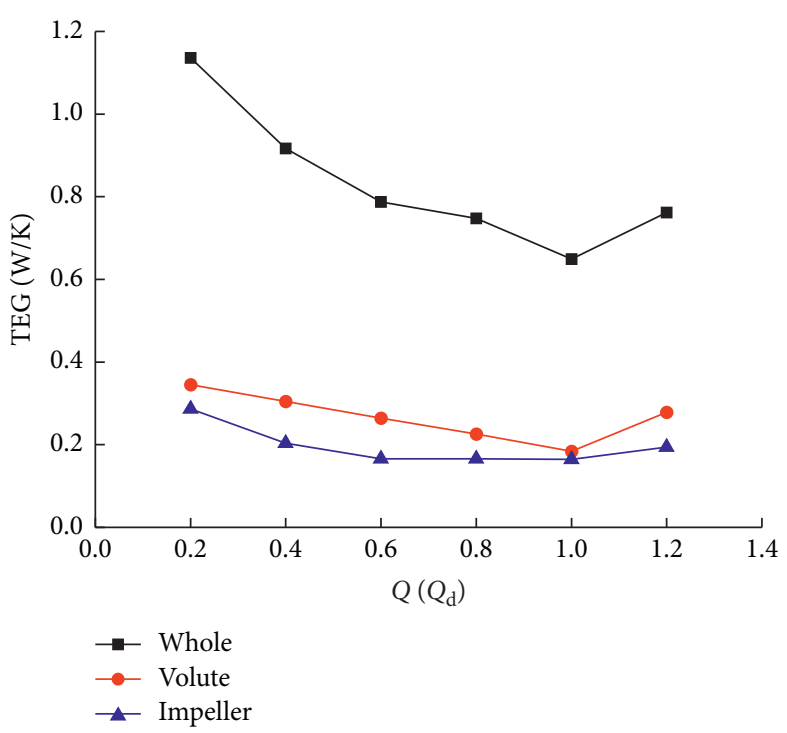

(b)

FIGURE 13: Distribution of total entropy generation. (a) Proportion of TEG for each component. (b) TEG for main flow parts.

$$
\mathrm{TEG}=\dot{S_{D}}=\int{ }_{V} S_{D}^{\prime \prime \prime} \mathrm{d} V
$$

3.5.1. Total Entropy Generation Rate Analyses. The distributions of total entropy generation at different flow rates are shown in Figure 9, where Figure 13(a) presents the proportion of TEG for each component including clearance, inlet, volute, and impeller domain, and Figure 13(b) presents the TEG of main flow components. From Figures 5 and 13, it can be seen that the TEG of whole domain and the efficiency of the centrifugal pump have opposite rule with the change of flow rate. The greater the total entropy generation value, the greater the energy loss and the lower the efficiency of the centrifugal pump. The change rule of vibration energy with flow rate on the impeller and the volute is consistent with that of total entropy generation. The vibration is a result of the poor flow conditions within the pump and it has an influence on the energy loss in centrifugal pump.

From Figure 13(a), it can be seen that the clearances of impeller side room result in greater energy loss and its proportion is about $40 \%$ at $1.0 Q_{\mathrm{d}}$. Also, the energy conversion of the volute leads to larger energy loss and its proportion is $37 \%$ at $1.2 Q_{\mathrm{d}}$. From Figure $13(\mathrm{~b})$, it can be seen that the maximum TEG value of whole domain, volute domain, and impeller domain is $1.14 \mathrm{~W} / \mathrm{K}, 0.35 \mathrm{~W} / \mathrm{K}$, and $0.29 \mathrm{~W} / \mathrm{K}$ at $0.2 Q_{\mathrm{d}}$, respectively. This is due to a series of unsteady flow in the centrifugal pump at low flow rate conditions, especially near the volute tongue, resulting in large energy loss. The TEG value of the volute domain is higher than that of the impeller domain. From $0.2 Q_{d}$ to $1.0 Q_{\mathrm{d}}$, the TEG value decreases with the increasing of the flow rate, and the TEG values in impeller domain decrease slightly at $0.6 Q_{\mathrm{d}}, 0.8 Q_{\mathrm{d}}$, and $1.0 Q_{\mathrm{d}}$. At design flow rate, the TEG reaches a minimum value of $0.65 \mathrm{~W} / \mathrm{K}, 0.18 \mathrm{~W} / \mathrm{K}$, and $0.16 \mathrm{~W} / \mathrm{K}$ in whole domain, volute domain, and impeller domain, respectively. From $1.0 Q_{\mathrm{d}}$ to $1.2 Q_{\mathrm{d}}$, the TEG values have a small increase, indicating that the energy loss increases with the increasing of flow rate, and the efficiency of centrifugal pump decreases. The attack angle of inflow increases as the flow rate increases, also it decreases as the flow rate decreases. They both cause impact loss to the surface of the blade.

3.5.2. Entropy Generation Rate Distribution. The vibration is a result of the poor flow patterns and consequently high losses in the pump. The operating points of $0.2 \mathrm{Qd}$ and 1.0Qd are selected as objects for EGR and streamline analysis in the impeller. Figure 14 shows the EGR and streamline distribution in the impeller on different spanwise surfaces (Span 0.4, Span 0.6, and Span 0.8) at $0.2 Q d$ and 1.0Qd. Span 0.4 is the surface close to the hub, and Span 0.8 is close to the shroud. At $0.2 Q_{\mathrm{d}}$, the energy loss of the impeller is mainly concentrated on the inlet and outlet of the blade. Because the inlet flow angle is less than the inlet install angle of blade, flow separation occurs at the front edge of the blade suction surface and gradually diffuses into the outlet of the impeller in the passage, resulting in a great separation loss. At the outlet of the blade, there is a great deal of energy loss generated by the backflow at the outlet of the impeller. As the flow rate increases, inflow attack angle gradually decreases. At $1.0 Q_{\mathrm{d}}$, the flow separation phenomenon and impact loss on the inlet of the blade become weakened, and the energy loss is drastically reduced. 


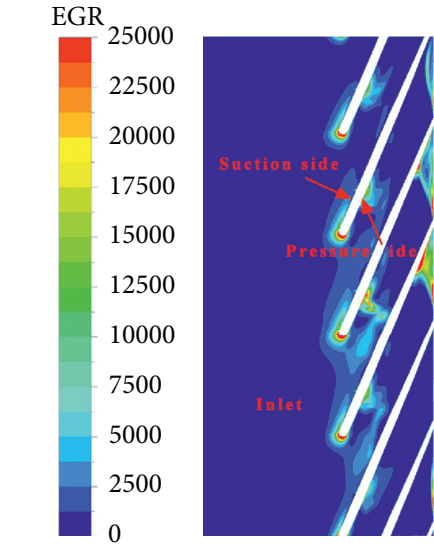

$\left[\mathrm{Wm}^{-3} \mathrm{~K}^{-1}\right]$

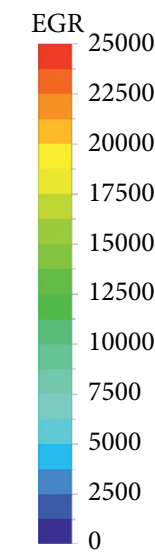

$\left[\mathrm{Wm}^{-3} \mathrm{~K}^{-1}\right]$
0.4 Span

(a)

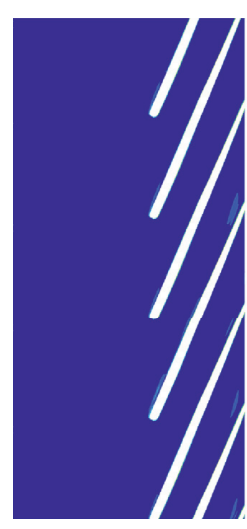

0.4 Span

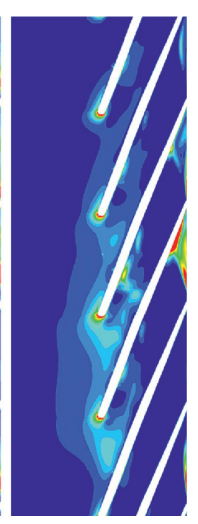

0.6 Span

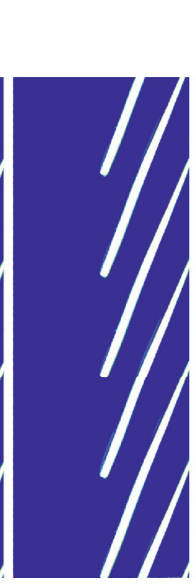

0.6 Span

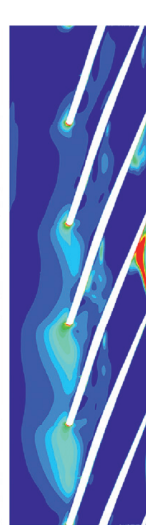

$0.8 \mathrm{Span}$

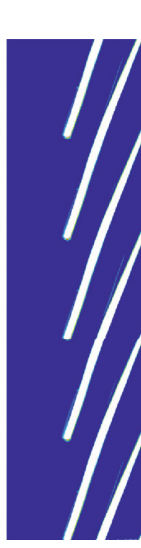

0.8 Span

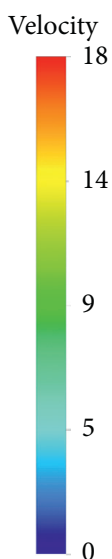

$\left[\mathrm{ms}^{-1}\right]$

14

9

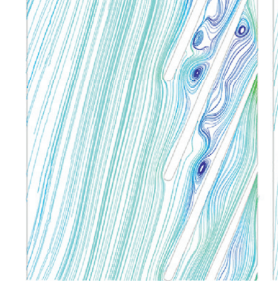

0.4 Span

(b)

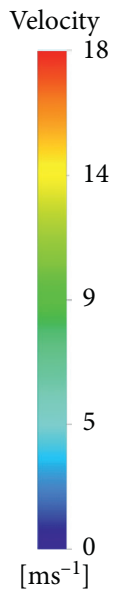

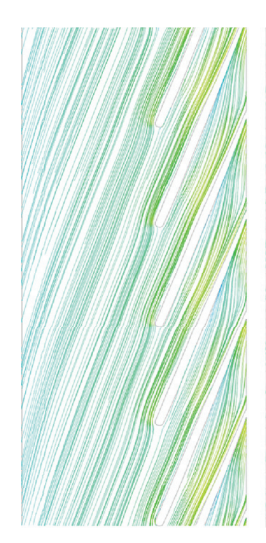

0.4 Span

(c)

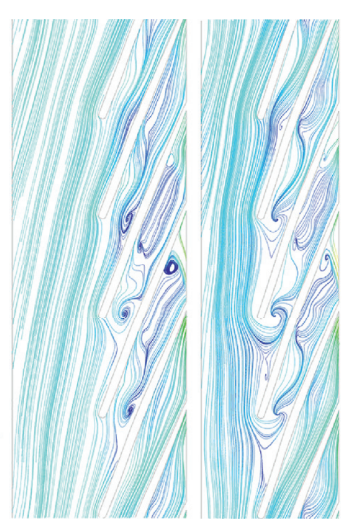

0.6 Span

0.8 Span

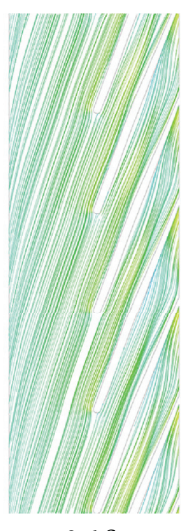

0.6 Span

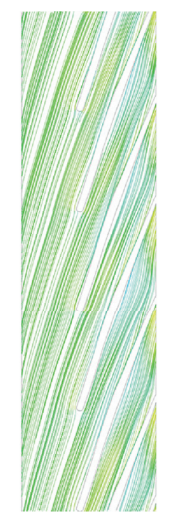

0.8 Span

(d)

FIGURE 14: EGR and streamline distribution in different spans of impeller. (a) EGR at $0.2 Q_{\mathrm{d}}$. (b) Streamline at $0.2 Q_{\mathrm{d}}$. (c) EGR at $1.0 Q_{\mathrm{d}}$. (d) Streamline at $1.0 Q_{\mathrm{d}}$.

\section{Conclusions}

Flow-induced impeller vibration and radial force in centrifugal pump were investigated through numerical analysis. And vibration energy and energy loss in the centrifugal pump were analyzed. The following conclusions can be drawn from the study:

(1) The radial force and vibration displacement of the impeller were lowest at the design flow rate and increased at off-design flow rates. The variation trend of the radial displacements in the $X$ - and $Y$-directions was consistent with that of the radial forces. The variation in the radial displacement lags that of the radial force.

(2) The TEG of whole domain and the efficiency of the centrifugal pump have opposite rule with the change of flow rate. The greater the total entropy generation value, the greater the energy loss and the lower the efficiency of the centrifugal pump.

(3) The change rules of vibration energy with flow rate on the volute and the impeller are consistent with that of total entropy generation of the volute and the impeller, respectively. The vibration is a result of the poor flow conditions within the pump, which have an influence on the energy loss in centrifugal pump.

(4) The higher EGR region is concentrated suction surface of front edge on the blade, pressure surface in the middle passage, and impeller outlet, where there is a great deal of energy loss generated by the rotorstator interaction, inflow impact, flow separation, backflow, and wake flow.

\section{Data Availability}

No data were used to support this study.

\section{Conflicts of Interest}

The authors declare that they have no conflicts of interest.

\section{Acknowledgments}

This work was supported by the Joint Project from the National Natural Science Foundation of China and Liaoning 
Province (Grant no. U1608258) and Zhejiang Provincial Natural Science Foundation of China (Grant no. LZ20E060002).

\section{References}

[1] R. Spence and J. Amaral-Teixeira, "A CFD parametric study of geometrical variations on the pressure pulsations and performance characteristics of a centrifugal pump," Computers \& Fluids, vol. 38, no. 6, pp. 1243-1257, 2009.

[2] R. Barrio, J. Parrondo, and E. Blanco, "Numerical analysis of the unsteady flow in the near-tongue region in a volute-type centrifugal pump for different operating points," Computers \& Fluids, vol. 39, no. 5, pp. 859-870, 2010.

[3] A. Fontanals, A. Guardo-Zabaleta, M. Coussirat, and E. Egusquiza, "Numerical study of the fluid-structure interaction in the diffuser passage of a centrifugal pump," Computational Methods for Coupled Problems Inence \& Engineering IV, vol. 30, no. 6, pp. 986-995, 2011.

[4] C. Münch, P. Ausoni, O. Braun, M. Farhat, and F. Avellan, "Fluid-structure coupling for an oscillating hydrofoil," Journal of Fluids and Structures, vol. 26, no. 6, pp. 1018-1033, 2010.

[5] R. L. Campbell and E. G. Paterson, "Fluid-structure interaction analysis of flexible turbomachinery," Journal of Fluids and Structures, vol. 27, no. 8, pp. 1376-1391, 2011.

[6] L. Zhang, Y. Guo, and W. Wang, "Large eddy simulation of turbulent flow in a true 3D Francis hydro turbine passage with dynamical fluid-structure interaction," International Journal for Numerical Methods in Fluids, vol. 54, no. 5, pp. 517-541, 2007.

[7] F.-K. Benra, "Numerical and experimental investigation on the flow induced oscillations of a single-blade pump impeller," Journal of Fluids Engineering, vol. 128, no. 4, pp. 783-793, 2005.

[8] K. Katsutoshi, O. Shigeyoshi, H. Ichiro, and Y. Chiba, "Numerical analysis of stress on pump blade by one-way coupled fluid-structure simulation," Journal of Fluid Science and Technology, vol. 5, no. 2, pp. 219-234, 2010.

[9] J. Pei, F. Meng, Y. Li, S. Yuan, and C. Jia, "Fluid-structure coupling analysis of deformation and stress in impeller of an axial-flow pump with two-way passage," Advances in Mechanical Engineering, vol. 8, no. 4, Article ID 168781401664626 , pp. 1-11, 2016.

[10] A. Schneider, B. C. Will, and M. Böhle, "Numerical evaluation of deformation and stress in impellers of multistage pumps by means of fluid structure interaction," in Proceedings of the ASME 2013 Fluids Engineering Division Summer Meeting, Nevada, USA, July 2013.

[11] J. Pei, F.-K. Benra, and H. Dohmen, "Application of different strategies of partitioned fluid-structure interaction simulation for a single-blade pump impeller," Proceedings of the Institution of Mechanical Engineers, Part E: Journal of Process Mechanical Engineering, vol. 226, no. 4, pp. 297-308, 2012.

[12] J. Pei, H. J. Dohmen, S. Q. Yuan, and F.-K. Benra, "Investigation of unsteady flow-induced impeller oscillations of a single-blade pump under off-design conditions," Journal of Fluids and Structures, vol. 35, pp. 89-104, 2012.

[13] Y. Ren, Z. Zhu, D. Wu, and X. Li, "Influence of guide ring on energy loss in a multistage centrifugal pump," Journal of Fluids Engineering, vol. 141, no. 6, Article ID 061302, 2019.

[14] X.-Q. Jia, Z.-C. Zhu, X.-L. Yu, and Y.-L. Zhang, "Internal unsteady flow characteristics of centrifugal pump based on entropy generation rate and vibration energy," Proceedings of the Institution of Mechanical Engineers, Part E: Journal of Process Mechanical Engineering, vol. 233, no. 3, Article ID 095440891876528, pp. 456-473, 2018.

[15] A. Bejan, Entropy Generation through Heat and Fluid Flow, John Wiley \& Sons, Hoboken, NJ, USA, 1982.

[16] F. Kock and H. Herwig, "Local entropy production in turbulent shear flows: a high-Reynolds number model with wall functions," International Journal of Heat and Mass Transfer, vol. 47, no. 10-11, pp. 2205-2215, 2004.

[17] J. Mathieu and J. Scott, An Introduction to Turbulent Flow, Cambridge University Press, Cambridge, United Kingdom, 2000. 